\title{
PIERCING POINTS OF CRUMPLED CUBES
}

\author{
BY \\ L. D. LOVELAND
}

McMillan [20] proved that a free 2-sphere in $S^{3}$ can be pierced by a tame arc at each of its points. Since each complementary domain of a free 2-sphere is an open 3-cell [22], it seems natural to attempt to prove some theorem analogous to McMillan's with this weaker hypothesis. We show that a crumpled cube $C$ in $S^{3}$ has at most one nonpiercing point if Int $C$ is an open 3-cell. A crumpled cube $C$ is the union of a 2-sphere and one of its complementary domains in $S^{3}$, and a point $p$ of $\mathrm{Bd} C$ is a piercing point of $C$ if there is a homeomorphism $h$ of $C$ into $S^{3}$ such that $h(\mathrm{Bd} C)$ can be pierced by a tame arc at $h(p)$. It follows that a 2 -sphere $S$ in $S^{3}$ has at most two points where $S$ cannot be pierced by a tame arc if each component of $S^{3}-S$ is an open 3-cell (Corollary 3). McMillan [21] obtained the same result independently using an entirely different approach. Our proof follows from Lemmas 1-3 and the main result of [8].

Other results follow from the methods used in the proofs of Lemmas 1-3. For example, if a Cantor set $W$ lies in a 2-sphere $S$ in $S^{3}$ such that each component of $S^{3}-S$ is an open 3-cell, then $W$ is tame (Theorem 3 ). Thus each Cantor set on a free 2-sphere is tame. We also show that a continuum $F$ is cellular if $F$ lies in the boundary $S$ of a cellular 3-cell and $F$ does not separate $S$ (Theorem 4).

We also obtain two characterizations of piercing points of crumpled cubes. One that is useful in this paper is that a point $p$ in the boundary $S$ of a crumpled cube $C$ is a piercing point of $C$ if and only if Property $(*, p$, Int $C$ ) is satisfied (Theorem 2). If $F$ is a closed subset of $S,(*, F$, Int $C)$ is defined to mean that Bing's Side Approximation Theorem [6] can be applied relative to $S$ and Int $C$ in such a way that the intersection with $S$ of the polyhedral approximation to $S$ lies in the union of a finite set of mutually disjoint small disks in $S-F$. A precise definition can be found in [11] or [15]. The other characterization of piercing points (see Corollary 4) follows as a consequence of this one and results from [17].

The references should be consulted for needed definitions.

\section{Piercing points of the closure of an open 3-cell.}

LEMMA 1. If $\varepsilon>0$ and $p$ and $q$ are points in the boundary $S$ of a crumpled cube $C$ in $S^{3}$, then there exist a crumpled cube $M$, an $\varepsilon$-homeomorphism $h$ taking $S$ onto Bd $M$, and a Sierpinski curve $X$ such that

(1) $p$ and $q$ are inaccessible points of $X$,

(2) $X \subset S \cap \mathrm{Bd} M$,

Received by the editors September 12, 1967. 
(3) $\mathrm{Bd} M$ is locally tame modulo $\{p, q\}$,

(4) $M$ lies in an $\varepsilon$-neighborhood of $C$,

(5) $h$ is the identity on $X$, and

(6) each component of $S-X$ has diameter less than $\varepsilon$.

Proof. We shall use the technique introduced by Martin [18]. Let $D$ be a disk on $S$ such that $p \in \operatorname{Int} D, q \in S-D$, and $\mathrm{Bd} D$ is tame [4], and let $J_{1}, J_{2}, \ldots$ be a sequence of tame simple closed curves in $D$ such that $\mathrm{Bd} J_{1}=\mathrm{Bd} D$ and if $D_{1}, D_{2}, \ldots$ are the disks on $D$ bounded by $J_{1}, J_{2}, \ldots$, respectively, then $D_{i+1} \subset D_{i}$ and $p=\bigcap D_{i}$. Using repeatedly the results from [6] and [11] together with the techniques of [4], we obtain a collection of tame annuli $A_{1}, A_{2}, \ldots$ such that

$\mathrm{Bd} A_{i}=\mathrm{Bd} D_{i} \cup \operatorname{Bd} D_{i+1}$,

$A_{i} \cap D_{i}$ contains a Sierpinski curve $X_{i}$ in $D_{i}-$ Int $D_{i+1}$,

$A_{j} \cap$ Int $A_{i}=\varnothing$ if $i \neq j$,

$\mathrm{Cl}\left(\cup A_{i}\right)$ is a disk $E$ with boundary $J_{1}$,

Bd $M=(S-D) \cup E$, and each component of $A_{i}-X_{i}$ has diameter less than $\varepsilon / i$.

The procedure for obtaining the annuli $A_{i}$ is given roughly by Martin in [18], so we do not pursue the details.

The same procedure as outlined above relative to $q$ and the disk $S-$ Int $D$ yields a sequence of Sierpinski curves $Y_{i}$ such that $\{p, q\} \cup\left(\cup X_{i}\right) \cup\left(\cup Y_{i}\right)$ is the Sierpinski curve $X$ required in Lemma 1.

Lemma 2. If the closure of $S^{3}-C$ is a 3-cell, then, in addition to the conditions in Lemma 1, $M$ and $X$ can be selected such that

(7) $C \subset M$,

(8) $\mathrm{Bd} M \cap S=X$, and

(9) the closure of each component of $M-C$ is a 3-cell.

Proof. We use Lemma 1 to obtain a Sierpinski curve $X$ such that $X$ contains $p$ and $q$ inaccessibly, $X$ is locally tame modulo $\{p, q\}$, and each component of $S-X$ is small. Since $S$ is tame from $S^{3}-C$ we can obtain a 2-sphere Bd $M$ by pushing each component of $S-X$ slightly into $S^{3}-C$. Since Bd $M$ is locally tame modulo $X$, we see that $\mathrm{Bd} M$ is locally tame modulo $\{p, q\}$ [5]. If we identify $M$ as the crumpled cube containing $C$ and bounded by $\mathrm{Bd} M$, conditions (7) and (8) follow. If $Z$ is the closure of a component of $M-C$, then $\mathrm{Bd} Z$ is a 2-sphere that is locally tame from Int $Z$ modulo a tame simple closed curve in $X$. It follows that $\operatorname{Bd} Z$ is tame from Int $Z$ (see [11, Theorem 2] and [15, Theorem 14]), so $Z$ is a 3-cell.

Lemma 3. Suppose $K$ is a crumpled cube in $S^{3}$ such that $S^{3}-K$ is an open 3-cell. If $K_{1}, K_{2}, \ldots$ is a sequence of mutually disjoint 3-cells in $K$ such that, for each $i$, $K_{i} \cap \mathrm{Bd} K$ is a disk $D_{i}$, then $S^{3}-\mathrm{Cl}\left(K-\bigcup_{1}^{\infty} K_{i}\right)$ is an open 3-cell.

Proof. For each integer $n$ we let $M_{n}=\mathrm{Cl}\left(K-\bigcup_{i=1}^{n} K_{i}\right)$ and $V_{n}=S^{3}-M_{n}$. The theorem will follow from [7] once we show that each $V_{n}$ is an open 3-cell or equivalently that each $M_{n}$ is cellular. In the remainder of the proof we show that 
$M=M_{1}$ is cellular. Since the $K_{i}$ are disjoint the same procedure can be used inductively to show the cellularity of each $M_{n}$.

Let $\alpha>0$ and let $A$ be a polyhedral arc in $S^{3}-K_{1}$ from a point $k$ in Int $K-K_{1}$ to a point $m$ in $S^{3}-K$. Since $K_{1}$ is a 3-cell, there exist disks $D$ and $E$ such that $D \subset$ Int $D_{1}$, Bd $D$ is tame, $\mathrm{Bd} D=\mathrm{Bd} E, E-\mathrm{Bd} E$ lies in Int $K_{1}$, and $E$ is homeomorphically within $\alpha / 2$ of $\mathrm{Bd} K_{1}-$ Int $D_{1}$. Using Bing's Side Approximation Theorem [6] relative to the open set $\mathrm{Bd} K-D$ in $\mathrm{Bd} K$, we obtain an annulus $F$ such that $\mathrm{Bd} D \subset \mathrm{Bd} F, \mathrm{Bd} F-\mathrm{Bd} D \subset S^{3}-K, F \cap(A \cup M \cup \operatorname{Int} E \cup$ Int $D)=\varnothing$, $F$ lies within $\alpha / 2$ of $M$, and $F$ is locally polyhedral modulo Bd $D$. Without loss in generality we may assume that $G=F \cup E$ is a polyhedral disk near $M$ [10].

Since $S^{3}-K$ is an open 3-cell, there is a 2-sphere $R$ within $\alpha$ of $\operatorname{Bd} K$ such that $R$ separates the two boundary components of $F, R$ separates $k$ from $m$, and $R$ lies in $S^{3}-K$. We assume that $R$ is polyhedral [1] and that $F$ and $R$ are in general position. Now we assume that $A$ and $R$ are in general position (i.e., $R \cap A$ consists of a finite number of points where $A$ pierces $R$ ), and we choose a component $T$ of $R-F$ such that $T \cap A$ consists of an odd number of points. Then $T$ is a disk with holes lying within $\alpha$ of $M$. We fill these holes with disks near $G$ to obtain a 2-sphere $W$ that lies within $\alpha$ of $M$. Since $A \cap W=A \cap T$, it follows that $W$ separates $k$ from $m$. This means that $W$ bounds a 3-cell $X$ such that $M \subset$ Int $X$ and $X$ lies within $\alpha$ of $M$.

THeOREM 1. If $C$ is a crumpled cube in $S^{3}$ such that Int $C$ is an open 3-cell, then $C$ has at most one nonpiercing point.

Proof. Since there is a homeomorphism $h$ of $C$ into $S^{3}$ such that the closure of $S^{3}-h(C)$ is a 3-cell [12], [13] and $p$ is a piercing point of $C$ if and only if $p$ is a piercing point of $h(C)$, we assume without loss in generality that the closure of $S^{3}-C$ is a 3-cell $K$. Let $p$ and $q$ be two points of $\mathrm{Bd} C$. We shall show that one of these two points must be a piercing point of $C$. It follows from Lemma 2 that there exists a 2-sphere $S^{\prime}$, a Sierpinski curve $X$, and a crumpled cube $M$ such that $M \subset K$, Bd $M=S^{\prime}, p$ and $q$ are inaccessible points of $X, S^{\prime} \cap \mathrm{Bd} C=X, S^{\prime}$ is locally tame modulo $\{p, q\}$, and the closure of each component of $K-M$ is a 3-cell. From Lemma 3 we see that $S^{3}-M$ is an open 3-cell. Since $K$ is a 3-cell it follows that $\mathrm{Bd} M$ is locally tame from Int $M$ at both $p$ and $q$ (in fact, $M$ is a 3-cell). For more detail, see the proof of Lemma 5 to follow.

It follows from [8, Theorem 1] that $\mathrm{Bd} M$ is also locally tame from $S^{3}-M$ at one of the points $p$ and $q$, say $p$. Then $p$ lies in a tame arc in $X$, so $p$ is a point at which $\mathrm{Bd} C$ can be pierced by a tame arc [11]. This means that $p$ is a piercing point of $C$.

Property $(*, p, \operatorname{Int} C)$, which is used in the following theorem, was defined roughly in the introduction and can be found in either [11] or [15].

THEOREM 2. A point $p$ in the boundary of a crumpled cube $C$ is a piercing point of $C$ if and only if $(*, p$, Int $C)$ is satisfied. 
Proof. If $p$ is a piercing point of $C$, there exists a homeomorphism $h$ of $C$ into $S^{3}$ such that $h(S)$ can be pierced by a tame arc at $h(p)$. According to Gillman [11] this means that $h(p)$ lies in a tame arc $A$ in $h(S)$. Furthermore Gillman [11] proved that $(*, A, h($ Int $C))$ is satisfied since $A$ is tame. Lister [14] showed that $\left(*, h^{-1}(A)\right.$, Int $C$ ) follows since $h$ is a homeomorphism. Of course this implies $(*, p$, Int $C)$.

The other half of the proof of Theorem 2 is merely a rearrangement of the same ideas.

The following result is a consequence of Theorem 2 and a result by Martin [19].

COROLLARY 1. If $p$ is a point in a 2-sphere $S$ in $S^{3}$ and $U$ and $V$ are the components of $S^{3}-S$, then either $(*, p, U)$ or $(*, p, V)$ is satisfied.

Corollary 2. If $C$ and $L$ are the crumpled cubes bounded by a 2-sphere $S$ in $S^{3}$ and a point $p \in S$ is a piercing point of both $C$ and $L$, then $S$ can be pierced by a tame arc at $p$.

Proof. It follows from Theorem 2 that both $(*, p$, Int $C)$ and $(*, p$, Int $L)$ are satisfied. This means that $S$ can be pierced by a tame arc at $p$ [11].

COROLlaRY 3. If each complementary domain of a 2-sphere $S$ in $S^{3}$ is an open 3-cell, then $S$ contains two points $p$ and $q$ such that $S$ can be pierced by a tame arc at each point of $S-\{p, q\}$.

Corollary 4. A point $p$ in the boundary of a crumpled cube $C$ is a piercing point of $C$ if and only if p lies in an arc $A$ in $\mathrm{Bd} C$ such that for each $\varepsilon>0$ there is a positive number $\delta$ such that each unknotted simple closed curve that lies in Int $C$ and has diameter less than $\delta$ can be shrunk to a point in an $\varepsilon$-subset of $S^{3}-A$.

Proof. From Theorem 2 we see that $(*, p$, Int $C)$ is satisfied if $p$ is a piercing point of $C$. Then $p$ lies in an arc $A$ satisfying the conditions of Corollary 4 (see Theorem 1 in [17] and the remark on p. 511 in [15]).

If there exists an $\operatorname{arc} A$ as in the statement of Theorem 2 , then $(*, A$, Int $C)$ follows (see the remark prior to the statement of Theorem 2 in [17]). Thus it follows from Theorem 2 that $p$ is a piercing point of $C$.

II. Certain Cantor sets are tame. Using the methods of $\S I$, we show that Cantor set $W$ is tame if $W$ lies in a 2 -sphere $S$ such that each component of $S^{3}-S$ is an open 3-cell.

LEMMA 4. If $\varepsilon>0$ and $W$ is a closed 0-dimensional subset of a 2-sphere $S$ in $S^{3}$, then there exists a Sierpinski curve $X$ in $S$ such that

(1) $W$ lies inaccessibly in $X$,

(2) $X$ is locally tame modulo $W$, and

(3) each component of $S-X$ has diameter less than $\varepsilon$.

Proof. The proof is much the same as that given for Lemma 1 . We let $B_{1}$ be a finite collection of mutually disjoint disks $D_{11}, D_{12}, \ldots, D_{1 n_{1}}$ in $S$ such that each 
Bd $D_{1 j}$ is tame, $W \subset \bigcap$ Int $D_{1 j}$, and diam $D_{1 j}<1$. We inductively define, for each positive integer $n$, a similar finite collection $B_{n}$ of disjoint disks each of diameter less than $1 / n$. If we denote the union of the disks in $B_{n}$ by $B_{n}^{*}$, then we insist that $B_{n}^{*}$ is in the interior of $B_{n-1}^{*}$ and that each point of $W$ is a component of $\bigcap_{n=1}^{\infty} B_{n}^{*}$.

Now we apply the procedure outlined in the proof of Lemma 1. First we obtain a tame Sierpinski curve $X_{1}$ in $S-\bigcup$ Int $D_{1 i}$ such that each component of $S-X_{1}$ has diameter less than $\varepsilon$. Then $X_{2}$ is a finite collection of tame Sierpinski curves each in the closure of a component of $B_{1}^{*}-B_{2}^{*}$ and having small holes. This process is continued so that, for each $n$, we obtain a finite collection $X_{n}$ of tame Sierpinski curves in the closure of $B_{n}^{*}-B_{n+1}^{*}$. If we denote the union of the Sierpinski curves in $X_{n}$ by $X_{n}^{*}$, then $\left(\cup X_{n}^{*}\right) \cup W$ is the desired Sierpinski curve $X$.

LemMA 5. If $W$ is a closed 0-dimensional subset of the boundary $S$ of a cellular 3-cell $C$ in $S^{3}$ and $X$ is a Sierpinski curve in $S$ containing $W$ such that $X$ is locally tame modulo $W$, then there exists a cellular 3-cell $M$ such that

(1) $\mathrm{Bd} M$ is locally tame modulo a point of $W$,

(2) $X=\mathrm{Bd} M \cap S$, and

(3) $M \subset C$.

Proof. The proof of Lemma 2 shows how to construct a crumpled cube $M$ satisfying conditions (1) through (3), and it follows from Lemma 3 that $M$ is cellular. Since $M \subset C$ and Int $C$ is 1-ULC it is not difficult to show that for each $\varepsilon>0$ there exists a $\delta>0$ such that each simple closed curve lying in Int $M$ and having diameter less than $\delta$ can be shrunk to a point in an $\varepsilon$-subset of Int $C$. This implies Property $(A, X$, Int $M)$, defined in [15], which is equivalent to $(*, X$, Int $M)$ [15, Theorems 8-10]. Hence $M$ is a 3-cell [15, Theorem 14]. It follows from [8] that $\mathrm{Bd} M$ is locally tame modulo a point of $W$.

THEOREM 3. If $W$ is a closed 0-dimensional subset of a 2-sphere $S$ in $S^{3}$ and each component of $S^{3}-S$ is an open 3-cell, then $W$ is tame.

Proof. Let $U$ and $V$ be the components of $S^{3}-S$, and let $X$ be a Sierpinski curve satisfying the conditions of Lemma 4 relative to some $\varepsilon>0$. There is a homeomorphism $h$ of $S \cup V$ into $S^{3}$ such that $S^{3}-h(V)$ is a cellular 3-cell [12], [13]. Since $h(X)$ is locally tame modulo $h(W)$ it follows from Lemma 5 that $h(X)$ is locally tame modulo a point $h(p) \in h(W)$. Each Sierpinski curve $h(Y)$ in $h(X)-h(p)$ satisfies $(*, h(Y), h(V))$ since $h(Y)$ is tame [11], so it follows from a result by Lister [14] that $(*, Y, V)$ is satisfied.

Applying the same argument, where $f$ is a homeomorphism of $S \cup U$ into $S^{3}$, we obtain a point $q \in W$ such that each Sierpinski curve $Y$ in $X-\{p, q\}$ satisfies both $(*, Y, V)$ and $(*, Y, U)$. Then $Y$ is tame [15]. This means that $X$ is locally tame modulo two points, so $W$ is locally tame modulo two points. A theorem proven by Bing [3] shows $W$ to be tame. 
COROllaRY 5. If a closed 0-dimensional set $W$ lies in the interior of a cellular disk $D$ in $S^{3}$, then $W$ is tame.

Proof. For each point $p \in W$ there exists a disk $D_{p}$ in Int $D$ and a 2-sphere $S_{p}$ such that $p \in \operatorname{Int} D_{p} \subset D_{p} \subset S_{p}$ and $S_{p}$ is locally tame modulo $D_{p}$ [2, Theorem 5]. It follows from the fact that $D_{p}$ is cellular [21] that each component of $S^{3}-S_{p}$ is an open 3-cell [23]. This means that $W$ is locally tame (Theorem 3 ), so $W$ is tame [3].

COROLlary 6. If $W$ is a closed 0-dimensional subset of a free 2-sphere $S$ in $S^{3}$, then $W$ lies in a tame Sierpinski curve on $S$.

Proof. The proof of Theorem 3 shows that $W$ lies in a Sierpinski curve $X$ in $S$ such that $X$ is locally tame modulo two points (each component of a free 2-sphere must be an open 3-cell [22]). Since these two points lie in tame arcs in $S$ (see [20, Theorem 5] and [11, Theorem 6]), they each lie in tame arcs in $X$ [11, Lemma 6.1]. Thus $X$ is tame [10].

\section{Other related results.}

LEMMA 6. If $\varepsilon>0$ and $F$ is a continuum in the boundary $S$ of a crumpled cube $C$ in $S^{3}$ such that $F$ does not separate $S$, then there exists a null sequence of mutually disjoint $\varepsilon$-disks $\left\{E_{i}\right\}$ in $S-F$ and a crumpled cube $M$ such that

(1) $F \cup\left(S-\cup\right.$ Int $\left.E_{i}\right) \subset \mathrm{Bd} M$,

(2) $\mathrm{Bd} M$ is locally tame modulo $F$, and

(3) $M$ lies in an e-neighborhood of $C$.

Proof. Since $F$ does not separate $S$ there is a sequence $\left\{D_{i}\right\}$ of disks on $S$ such that $F=\bigcap D_{i}, \operatorname{Bd} D_{i}$ is tame, and $D_{i+1} \subset \operatorname{Int} D_{i}$. Now we follow the procedure outlined in the proof of Lemma 1 .

LemMA 7. If $\varepsilon>0$ and $F$ is a continuum in the boundary $S$ of a cellular 3-cell $C$ in $S^{3}$ such that $F$ does not separate $S$, then there exists a cellular 3-cell $M$ satisfying all the conditions of Lemma 6 and such that $M \subset C$.

Proof. We use Lemma 6 to obtain the disks $E_{i}$ in $S-F$. Then the construction of $M$ is indicated in the proofs of Lemmas 2 and 3 where each $E_{i}$ is replaced with a tame disk in $C$. Since $M \subset C$ and $C$ is a 3-cell it is easy to see that $M$ is also a 3-cell (see the proof of Lemma 5).

THEOREM 4. If $F$ is a continuum on the boundary $S$ of a cellular 3-cell in $S^{3}$ such that $F$ does not separate $S$, then $F$ is cellular.

Proof. It follows from Lemma 7 that there exists a cellular 3-cell $M$ such that Bd $M$ is locally tame modulo $F$. Then Theorem 5.2 of [23] insures that $F$ is cellular.

REMARK. The hypothesis that $S$ is the boundary of a 3-cell in the previous theorem cannot be removed. Furthermore, Theorem 4 becomes false if we require 
only that each component of $S^{3}-S$ be an open 3-cell. For example, one can grow two of the "feelers" described in [9] into opposite complementary domains of a sphere and let $F$ be any arc containing the two wild points of the resulting sphere. It then follows from [16] that $F$ is not cellular. However, $F$ ought to be cellular if each component of $S^{3}-S$ is an open 3-cell and $F$ contains at most one (of the two possible) points where $S$ cannot be pierced by a tame arc. Theorem 5 is a special case of this conjecture.

THEOREM 5. If $S$ is a 2-sphere in $S^{3}$ that is locally tame modulo a 0 -dimensional set, $F$ is a subcontinuum of $S$ that does not separate $S$, each component of $S^{3}-S$ is an open 3-cell, and $F$ contains at most one of the two possible points where $S$ cannot be pierced by a tame arc, then $F$ is ce!!ular.

Proof. Under the conditions of the hypothesis $S$ can have at most two wild points [8] and $F$ can contain at most one of these points. Then there exists a disk $D$ on $S$ and a point $p \in F$ such that $F \subset$ Int $D$ and $S$ is locally isme at each point of $D-p$. It follows from Corollary 1 and Theorem 14 of [15] that $S$ is locally tame from one component $V$ of $S^{3}-S$ at $p$. If $C=S \cup V$, then $C$ is a cellular crumpled cube. Now we are able to use the technique in the proofs of Lemmas 6 and 7 to obtain a cellular 3-cell $M$ such that $M \subset C, D \subset \mathrm{Bd} M$, and $\mathrm{Bd} M$ is locally tame modulo $D$. Then $\mathrm{Bd} M$ is locally tame modulo $F[10]$, and Theorem 5 follows from Theorem 4.

\section{REFERENCES}

1. R. H. Bing, Approximating surfaces with polyhedral ones, Ann. of Math. 65(1957), 456-483.

2. - A surface is tame if its complement is 1-ULC, Trans. Amer. Math. Soc. 101 (1961), 294-305.

3. - Tame Cantor sets in $E^{3}$, Pacific J. Math. 11 (1961), 435-446.

4. —- Each disk in $E^{3}$ contains a tame arc, Amer. J. Math. 84 (1962), 583-590.

5. - Pushing a 2-sphere into its complement, Michigan Math. J. 11 (1964), 33-45.

6. - Improving the side approximation theorem, Trans. Amer. Math Soc. 116 (1965), 511-525.

7. M. Brown, The monotone union of open n-cells is an open n-cell, Proc. Amer. Math. Soc. 12 (1961), 812-814.

8. C. E. Burgess, Criteria for a 2-sphere in $S^{3}$ to be tame modulo two points, Michigan Math. J. 14 (1967), 321-330.

9. R. H. Fox and E. Artin, Some wild cells and spheres in three-dimensional spaces, Ann. of Math. 49 (1948), 979-990.

10. P. H. Doyle and J. G. Hocking, Some results on tame disks and spheres in $E^{3}$, Proc. Amer. Math. Soc. 11 (1960), 832-836.

11. D. S. Gillman, Side approximation, missing an arc, Amer. J. Math. 85 (1963), 459-476.

12. N. Hosay, The sum of a real cube and a crumpled cube is $S^{3}$, Notices Amer. Math. Soc. 10 (1963), 668.

13. L. L. Lininger, Some results on crumpled cubes, Trans. Amer. Math. Soc. 118 (1965), 534-549.

14. F. M. Lister, Simplifying the intersection of disks in Bing's Side Approximation Theorem, Pacific J. Math. 22 (1967), 281-295. 
15. L. D. Loveland, Tame subsets of spheres in $E^{3}$, Pacific J. Math. 19 (1966), 489-517.

16. - Wild points of cellular subsets of 2-spheres in $S^{3}$, Michigan Math. J. 14 (1967), 427-431.

17. - Sufficient conditions for a closed set to lie on the boundary of a 3-cell, Proc. Amer. Math. Soc. 19 (1968), 649-652.

18. J. Martin, Tame arcs on disks, Proc. Amer. Math. Soc. 16 (1965), 131-133.

19. - The sum of two crumpled cubes, Michigan Math. J. 13 (1966), 147-151.

20. D. R. McMillan, Jr., Some topological properties of piercing points, Pacific J. Math. 22 (1967), 313-322.

21. —-, Piercing a disk along a cellular set, Proc. Amer. Math. Soc. 19 (1968), 153-157.

22. C. D. Papakyriakopoulos, On Dehn's lemma and the asphericity of knots, Ann. of Math. 66 (1957), 1-26.

23. D. G. Stewart, Cellular subsets of the 3-sphere, Trans. Amer. Math. Soc. 114 (1965), $10-22$.

\section{Utah State University, Logan, UTAH}

